\title{
Levels of Total Platelet Mass in Patients Admitted to Emergency Department and Diagnosed with Obstructive Sleep Apnea Syndrome
}

\author{
(1) Eyyüp Sabri Șeyhanlı1 ${ }^{1}$, (1) Ibrahim Halil Yasak²
}

1Department of Emergency Medicine, University of Health Sciences Turkey, Mehmet Akif İnan Training and Research Hospital, Șanlıurfa, Turkey

2Department of Emergency Medicine, Harran University Faculty of Medicine, Șanlıurfa, Turkey

\begin{abstract}
Aim: The objective of this study was to investigate the possibility of a relationship between the value of the platelet mass index and obstructive sleep apnea syndrome (OSAS) and to compare biochemical parameters among comorbidity groups in OSAS patients.

Materials and Methods: The patients were divided into two groups; the patient group consisted of patients who had previously presented to the emergency department of our hospital and were diagnosed with OSAS through polysomnography, while the control group included individuals who had no diagnosed chronic diseases.

The patients in the OSAS group were further divided into subgroups of patients with diabetes, diabetes + hypertension, diabetes + hyperlipidemia, diabetes + cardiovascular disease, diabetes + hypertension + hyperlipidemia, hyperlipidemia, cardiovascular disease, and hypertension.

Physical examination, complete blood count, and biochemical test outcomes of both groups as well as polysomnography data of the patient group were evaluated retrospectively.

Results: The mean BMI value was statistically significantly higher in OSAS patients than in with patients of the control group (24.28 \pm 1.00 vs $29.73 \pm 2.51 \mathrm{~kg} / \mathrm{m}^{2}$ ). The mean platelet volume (MPV) was significantly higher in the control group (10.13 \pm 1.09$)$ than in the OSAS group $(7.98 \pm 1.32)(p<0.001)$. The total platelet mass index, calculated by multiplying the platelet count by the MPV, was significantly higher in the control group $(2,737.92 \pm 548.90)$ than in the OSAS patient group $(2,104.89 \pm 462.86)(p<0.001)$

Conclusion: According to the results of this study, the platelet mass index, MPV, and/or total platelet count are not diagnostic. However, in patients presenting to the emergency department with complaints of fatigue, headache, inattention, and daytime drowsiness, they may be warning parameters indicating the possibility of OSAS.
\end{abstract}

Keywords: Emergency service, obstructive sleep apnea syndrome, platelet mass index

\section{Introduction}

Obstructive sleep apnea syndrome (OSAS) is a sleep-related respiratory disease characterized by repeating apnea and hypopnea episodes due to the obstruction of the airway. OSAS is associated with repeating nocturnal arterial oxygen desaturation and hypercapnia as well as with changes in systemic and pulmonary arterial pressure (1). The prevalence of OSAS has been reported as $4 \%$ in middle-aged men and $2 \%$ in middle-aged women (2). Moderate OSAS is seen in one of each five adults and moderate-to-severe OSAS in one of each fifteen adults (3). In OSAS, the interruption of the upper airway is defined as apnea, and decreased airway as hypopnea. These apnea and hypopnea cycles are repeated several times in every hour, causing fragmentation of sleep. Pharynx, and especially hypopharynx and oropharynx are the regions with obstructive processes that mostly lead to OSAS (4). OSAS is an independent risk factor for many diseases including heart failure, heart attack, cardiovascular events, and arrhythmias. OSAS has been associated with cardiovascular, growth and neurobehavioral anomalies and inflammation (5). 
Daytime drowsiness, decreased attention capacity, insufficient memory and learning, walking dysfunction, decreased motor performance and depressive symptoms may be seen in OSAS patients.

Platelets play an important role in the formation of atherosclerotic plaques and thus in the progression of atherosclerotic lesions to thrombosis. Platelets express and release substances involved in coagulation, inflammation, thrombosis and atherosclerosis processes $(6,7)$. In addition, platelets also play a role in the pathogenesis of coronary artery disease. Larger platelets are associated with increased enzymatic and metabolic activities, showing an increased prothrombotic condition (8). Mean platelet volume (MPV) and platelet distribution width (PDV) are the most commonly measured platelet indices during routine blood count. Studies have reported that the activation of platelets, MPV and PDW are increased in OSAS patients $(9,10)$. However, some studies have reported no association between platelet volume and OSAS (11). MPV should be interpreted with platelet count. Multiplying of MPV and platelet count is named as total platelet mass (12).

There are several studies in the literature about the relationship between platelet indices and OSAS (13-15). However, to our best knowledge the relation between platelet mass and OSAS has not been investigated previously. Therefore, the objective of this study was to investigate whether there was a relationship between the levels of platelet mass and OSAS and to compare biochemical parameters among comorbidity groups among OSAS patients.

\section{Materials and Methods}

\section{Study Population}

A total of 311 patients were included in the study with 208 who presented to the emergency department of our hospital with complaints such as fatigue, headache, distractibility and daytime drowsiness, and then were diagnosed with OSAS through polysomnography and 103 persons without the diagnosis of OSAS as the control group. Outcomes of the previously performed polysomnography of OSAS patients were evaluated together with a thoracic diseases specialist. Patients included in the study were divided into two groups as OSAS and non-OSAS patients. In addition, patients in the OSAS group were further divided according to their comorbidities into subgroups as diabetes, diabetes + hypertension, diabetes + hyperlipidemia, diabetes + cardiovascular disease, diabetes + hypertension + hyperlipidemia, hyperlipidemia, cardiovascular disease and hypertension. All patients underwent physical examination, electrocardiogram (ECG), complete blood count and biochemical laboratory analyses, while OSAS patients additionally underwent polysomnography.

Patients with a systolic blood pressure $>140 \mathrm{mmHg}$ or a diastolic blood pressure $>90 \mathrm{mmHg}$ or those receiving antihypertensive drugs were considered hypertensive. Patients with a fasting blood glucose $>126 \mathrm{mg} / \mathrm{dL}$ and those receiving antidiabetics were considered to have diabetes mellitus (DM). Patients aged under 18 years were excluded from the study.

\section{Polysomnography}

In this study, the diagnosis of OSAS was established with polysomnography which is accepted as the gold diagnostic method. All patients underwent a detailed nocturnal analysis with 44-channel polysomnography using the international 10-20 electrode insertion system.

Polysomnographic evaluation included electroencephalogram, electrooculogram with $256 \mu \mathrm{v} /$ hour sampling rate, submental electromyogram with 10-100 Hz and ECG with $0.2-30 \mathrm{~Hz}$ filtration. Respiratory effort was measured with abdominal and thoracic piezoelectric belts with $0.05-3 \mathrm{~Hz}$ filtration, while airflow was monitored with a nasal cannula. Oxygen saturation and pulse were measured using a finger pulse oximeter. In order to record leg movements, motion sensors of $1-20 \mathrm{~Hz}$ were inserted to each leg. Polysomnography processes were performed according to the Rechtschaffen and Kales criteria by two thoracic diseases specialists that showed $80-95 \%$ compliance to 50 reference data and had an experience of at least 1,000 polysomnography processes (16).

Interruption of airway at least for 10 seconds as a result of polysomnography was considered as apnea, while a decrease $\geq 3 \%$ in capillary oxygen saturation was considered as desaturation. Whereas, stimulation or desaturation lasting for $\geq 10$ seconds with a decreased air flow by 50\% during sleep was considered as hypopnea.

\section{Biochemical Measurements}

Blood samples were collected using a sterile syringe from the antecubital vein between a.m. 08:00 and 10:00 following 12-hour fasting. Glucose, creatinine and lipid profiles were determined with standard methods in the samples. MPV and platelet values were measured from the blood samples put into the dipotassium Ethylenediamine tetraacetic acid (EDTA) containing tubes. Complete blood count was performed using an automated blood counter (Beckman Coulter, Dx500, USA). MPV measurements were performed within 30 minutes after the sampling in order to prevent platelet swelling resulted from EDTA.

\section{Ethics Statement}

Before the beginning, the study protocol was approved by the local ethics committee of our hospital with 11/05/2020 dated 
and HRU/20.09.09 numbered decision. The study was conducted in accordance with the ethical principles of the Declaration of Helsinki.

\section{Statistical Analysis}

Data obtained in the study were statistically analyzed using SPSS for Windows version 23.0 package software (SPSS, IBM Inc., Statistical Package for Social Sciences, Illinois, Chicago, USA). Normality of the variables was analyzed with the Shapiro-Wilk test. In the comparison of the numerical data between the patient and control groups, one-way variance analysis (ANOVA) and Duncan multiple comparison tests were used for the normally distributed variables, and Kruskal-Wallis and Dunn multiple comparison tests for the non-normally distributed variables. Descriptive statistics are expressed as mean \pm standard deviation for numerical variables, while frequency and percentage were used for the categorical variables. $\mathrm{P}<0.05$ values were considered statistically significant.

\section{Results}

In this study, a total of 311 patients with 208 who presented to the emergency department of our hospital with complaints such as fatigue, headache, distractibility and daytime drowsiness, and then were diagnosed with OSAS through polysomnography and 103 persons without the diagnosis of OSAS were included as the control group. In addition, OSAS patients were divided into subgroups according to their comorbidities and the results of biochemical analysis and polysomnography were studied among these subgroups. Accordingly, DM was found in 34, diabetes + hypertension in 15 , diabetes + hyperlipidemia in 29 , diabetes + cardiovascular disease in nine, diabetes + hypertension + hyperlipidemia in 13, hyperlipidemia in 62, cardiovascular disease in 15 and hypertension in 31 patients. Distribution of the comorbidities in OSAS patients is given in Figure 1.

The mean age of the patients was $51.87 \pm 11.07$ (minimummaximum: 26-80) years. The mean age was $55.25 \pm 10.86$ years in the OSAS group and $46.85 \pm 9.23$ years in the control group. Accordingly, the mean age was statistically significantly higher in the OSAS group than in the controls $(p<0.001)$. The mean age was found to be statistically significantly higher in diabetes + cardiovascular group $(60.89 \pm 14.31)$ compared to cardiovascular disease $(60.47 \pm 11.60)$, diabetes + hypertension + hyperlipidemia

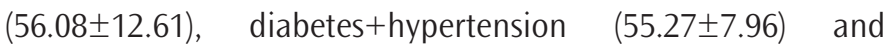
significantly higher in diabetes patients $(54.56 \pm 11.26)$ than in the control group (46.85 \pm 9.23$)$, diabetes + hyperlipidemia

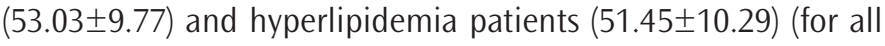
$\mathrm{p}<0.05)$.

The mean body mass index (BMI) value was statistically significantly higher in OSAS patients compared to the control group ( $24.28 \pm 1.00$ vs $\left.29.73 \pm 2.51 \mathrm{~kg} / \mathrm{m}^{2}\right)$. Examining according to the subgroups, no statistically significant difference was found in terms of BMI $(p>0.05)$.

Distribution of the demographic and clinical features is given in Table 1.

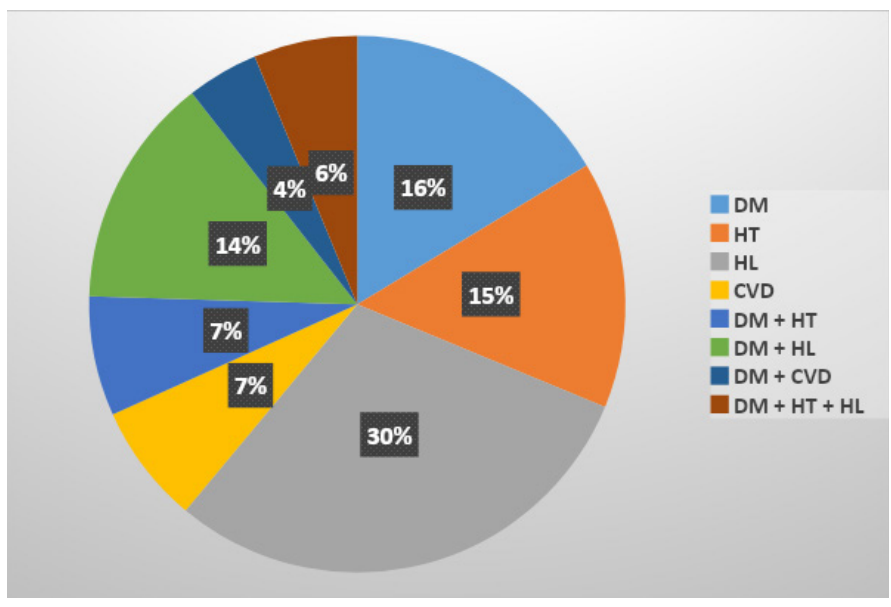

Figure 1. Distribution of the comorbidities in OSAS patients

OSAS: Obstructive sleep apnea syndrome, DM: Diabetes mellitus, HT: Hypertension, HL: Hyperlipidemia, CVD: Cardiovascular diseases

Table 1. Demographic and clinical characteristics of the patients

\begin{tabular}{|l|l|l|}
\hline & Mean \pm SD & Min-max \\
\hline Age & $51.87 \pm 11.07$ & $26.00-80.00$ \\
\hline BMI & $27.93 \pm 3.34$ & $21.97-36.93$ \\
\hline WBC & $9.06 \pm 2.30$ & $1.46-19.59$ \\
\hline Neutrophils & $5.27 \pm 1.85$ & $2.38-14.73$ \\
\hline Lymphocytes & $2.84 \pm 0.91$ & $0.32-6.17$ \\
\hline Monocytes & $0.68 \pm 0.22$ & $0.12-2.05$ \\
\hline RBC & $5.15 \pm 0.62$ & $3.18-7.02$ \\
\hline PLT & $269.34 \pm 61.18$ & $138.90-512.00$ \\
\hline MPV & $8.70 \pm 1.59$ & $5.36-12.30$ \\
\hline RDW & $12.74 \pm 1.64$ & $9.80-22.70$ \\
\hline Lymphocyte to monocyte & $4.56 \pm 2.18$ & $1.14-23.58$ \\
\hline Neutrophil to lymphocyte & $2.11 \pm 1.71$ & $0.78-25.91$ \\
\hline PLT x MPV & $2314.54 \pm 575.53$ & $1104.90-4710.40$ \\
\hline RDW/RBC & $2.51 \pm 0.53$ & $0.00-4.62$ \\
\hline PLT/RDW & $21.41 \pm 5.43$ & $10.37-39.75$ \\
\hline RDW/MPV & $1.51 \pm 0.35$ & $0.00-2.81$ \\
\hline Gender, n (\%) & \multicolumn{2}{|l}{} \\
\hline Male & $215(69.1)$ & - \\
\hline Female & $96(30.9)$ & - \\
\hline $\begin{array}{l}\text { BMI: Body mass index, WBC: White blood cells, RBC: Reb blood cells, PLT: Platelet, } \\
\text { MPV: Mean platelet volume, RDW: }: \text { Red cell distribution } \\
\text { Minimum, max: Maximum }\end{array}$ & \\
\hline & \multicolumn{2}{|l}{ Number, min: } \\
\hline
\end{tabular}


The MPV was significantly higher in the control group (10.13 \pm 1.09$)$ compared to the OSAS group $(7.98 \pm 1.32) \quad(p<0.001)$. Total platelet mass found with multiplying platelet counts and MPV was significantly higher in the control group $(2,737.92 \pm 548.90)$ compared to the OSAS patients $(2,104.89 \pm 462.86) \quad(p<0.001)$. Distribution of the demographic and clinical data of the participants is shown in Table 2.

\section{Discussion}

The association of OSAS with diseases such as cardiovascular disorders, hypertension and DM is well-known. Platelets are inflammatory markers playing a crucial role in the pathogenesis of numerous diseases, especially cardiovascular diseases (CVD). Larger platelets involve more granule and thromboxane $A 2$ and express more glycoproteins IB and IIB/IIIA receptors. Therefore, these large platelets lead to an increase in thromboembolic events by accumulating more rapidly (17-19).

There are several studies in the literature investigating the relationship between OSAS and MPV $(10,20,21)$. MPV is known to have an important role in the pathophysiology of CVD as an indicator of platelet activation (22). Since CVD increase in OSAS patients, studies in the literature have focused on the role of activated platelets. However, because as the size of platelets increases their effects on the above mentioned diseases increase, MPV should be interpreted together with platelet count. Total platelet mass is formulated as MPV $x$ platelet count. Platelet mass is an indicator of platelet function and activation (23). To our best knowledge, there is no study in the literature evaluating the effect of total platelet mass on OSAS.

Personal characteristics such as advanced age, hypertension and obesity are risk factors for OSAS and CVD (24). Structural changes like obesity predisposes OSAS patients to upper airway resistance and larger respiratory effort. In the present study, the mean age was statistically significantly higher among OSAS patients compared to the controls. In addition, when the subgroups were analyzed,

\begin{tabular}{|c|c|c|c|c|c|c|c|c|c|c|c|c|c|c|c|c|c|}
\hline$=$ & $\overline{8}$ & $\begin{array}{l}\bar{\delta} \\
\dot{0}\end{array}$ & 玄 & $\begin{array}{c}\infty \\
\stackrel{\infty}{m} \\
0\end{array}$ & $\begin{array}{l}\stackrel{0}{0} \\
\stackrel{0}{0}\end{array}$ & 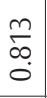 & $\begin{array}{l}\stackrel{\llcorner}{\widetilde{N}} \\
\stackrel{0}{0}\end{array}$ & $\mid \begin{array}{c}0 \\
0 \\
\infty \\
0 \\
0\end{array}$ & $\begin{array}{l}\bar{o} \\
\dot{0}\end{array}$ & $\stackrel{\widetilde{m}}{\circ}$ & $\begin{array}{l}0 \\
\vdots \\
0 \\
0\end{array}$ & 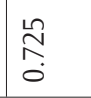 & $\bar{\delta}$ & $\begin{array}{l}0 \\
\stackrel{0}{0} \\
0\end{array}$ & $\stackrel{\infty}{\sigma}$ & $\bar{o}$ & \\
\hline 吉 & 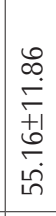 & 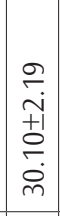 & 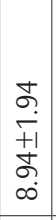 & 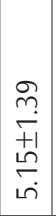 & $\begin{array}{c}\tilde{J} \\
0 \\
0 \\
+1 \\
\infty \\
\infty \\
\sim \\
\end{array}$ & $\begin{array}{l}0 \\
0 \\
+1 \\
01 \\
0 \\
0 \\
0\end{array}$ & 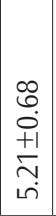 & 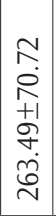 & 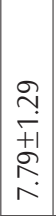 & 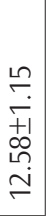 & $\begin{array}{l}\stackrel{゚}{2} \\
\stackrel{+}{+1} \\
m \\
\stackrel{r}{+}\end{array}$ & $\begin{array}{l}0 \\
0 \\
0 \\
+1 \\
0 \\
0 \\
i\end{array}$ & 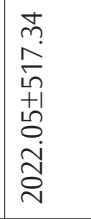 & 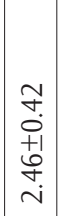 & 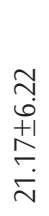 & $\begin{array}{l}m \\
\tilde{0} \\
0 \\
+1 \\
0 \\
0 \\
-\end{array}$ & \\
\hline ¿ & $\begin{array}{l}8 \\
\dot{\theta} \\
\dot{+} \\
+1 \\
\dot{f} \\
\dot{8}\end{array}$ & $\begin{array}{l}\bar{\sigma} \\
i \\
+1 \\
\overline{0} \\
\dot{\nu} \\
\end{array}$ & 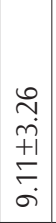 & 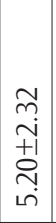 & 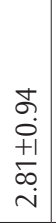 & 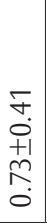 & 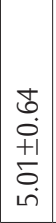 & 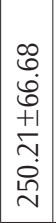 & 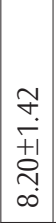 & 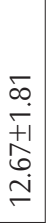 & 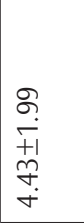 & 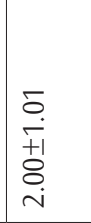 & 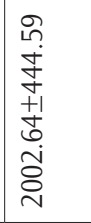 & 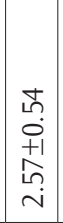 & 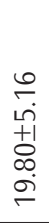 & 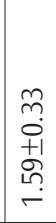 & \\
\hline 咅 & 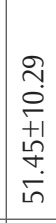 & 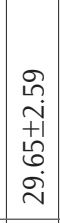 & 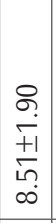 & 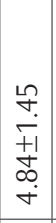 & 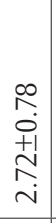 & 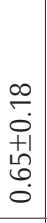 & 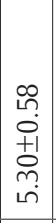 & 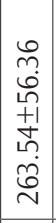 & $\begin{array}{c}\stackrel{2}{N} \\
⿱ 亠 䒑 \\
+1 \\
\vdots \\
\infty \\
\infty\end{array}$ & 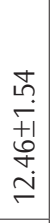 & $\begin{array}{l}\stackrel{N}{N} \\
\dot{+1} \\
\stackrel{+1}{f} \\
\dot{+} \\
\end{array}$ & $\begin{array}{l}\infty \\
\stackrel{0}{0} \\
+1 \\
+ \\
\sigma \\
\end{array}$ & 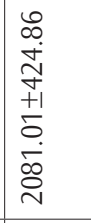 & $\begin{array}{l}0 \\
i \\
o \\
+1 \\
0 \\
0 \\
i \\
\end{array}$ & 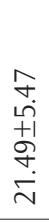 & 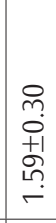 & \\
\hline 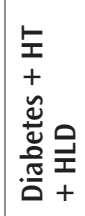 & $\begin{array}{l}\bar{\sigma} \\
\underset{1}{+1} \\
+1 \\
0 \\
0 \\
0 \\
\dot{0} \\
\llcorner\end{array}$ & 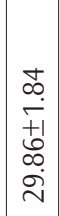 & 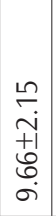 & 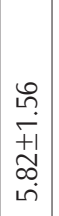 & 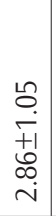 & 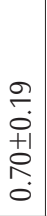 & $\begin{array}{c}8 \\
8 \\
0 \\
+1 \\
0 \\
\\
\text { ம் } \\
\end{array}$ & 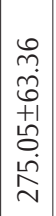 & 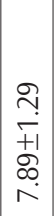 & 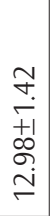 & $\begin{array}{l}\stackrel{f}{+} \\
\stackrel{i}{j} \\
+1 \\
\stackrel{+}{+} \\
\dot{+}\end{array}$ & $\begin{array}{l}\infty \\
\infty \\
0 \\
0 \\
+1 \\
0 \\
\stackrel{1}{1} \\
\end{array}$ & 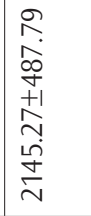 & $\begin{array}{l}+ \\
0 \\
0 \\
+1 \\
+ \\
0 \\
i\end{array}$ & 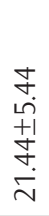 & 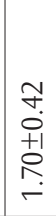 & \\
\hline 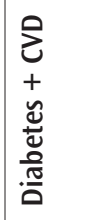 & $\begin{array}{l}\bar{n} \\
\dot{+} \\
+1 \\
\infty \\
\infty \\
\dot{8} \\
\dot{0}\end{array}$ & 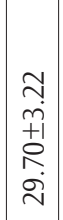 & $\mid \begin{array}{c}\stackrel{g}{+} \\
\stackrel{+}{+1} \\
0 \\
\infty \\
\infty \\
\end{array}$ & $\mid \begin{array}{c}\hat{a} \\
\dot{+} \\
+1 \\
o \\
+ \\
\dot{\sigma} \\
\dot{\sigma}\end{array}$ & $\begin{array}{l}\bar{\delta} \\
0 \\
0 \\
+1 \\
0 \\
0 \\
i\end{array}$ & 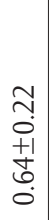 & \begin{tabular}{|c|}
0 \\
0 \\
0 \\
+1 \\
$\stackrel{1}{1}$ \\
$\dot{\sim}$ \\
\end{tabular} & 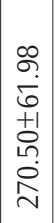 & 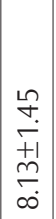 & 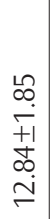 & 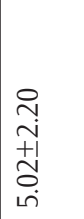 & $\begin{array}{l}0 \\
0 \\
0 \\
+1 \\
0 \\
0 \\
\stackrel{0}{-} \\
-1\end{array}$ & 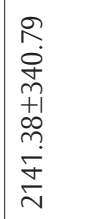 & $\mid \begin{array}{c}0 \\
0 \\
0 \\
+1 \\
0 \\
0 \\
i \\
\end{array}$ & $\begin{array}{l}\infty \\
m \\
\stackrel{\infty}{L} \\
+1 \\
\stackrel{+}{+} \\
\stackrel{\sim}{N}\end{array}$ & 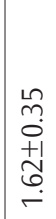 & \\
\hline 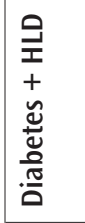 & $\begin{array}{l}\hat{\alpha} \\
\hat{\sigma} \\
+1 \\
0 \\
\hat{\sigma} \\
\hat{n}\end{array}$ & 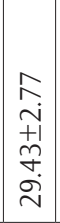 & $\begin{array}{c}\mp \\
\tilde{\sim} \\
+1 \\
\stackrel{1}{0} \\
\tilde{\sigma} \\
\end{array}$ & 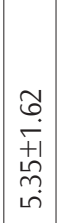 & $\begin{array}{c}\infty \\
\infty \\
0 \\
+1 \\
+ \\
\infty \\
i \\
ن\end{array}$ & 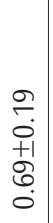 & 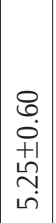 & 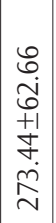 & 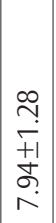 & 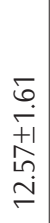 & $\begin{array}{l}\stackrel{g}{\sigma} \\
\stackrel{+}{+1} \\
\stackrel{9}{\sigma} \\
\dot{\sigma}\end{array}$ & 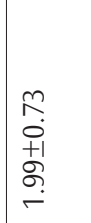 & 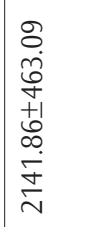 & $\mid \begin{array}{c}0 \\
\stackrel{0}{0} \\
+1 \\
+1 \\
\\
i\end{array}$ & 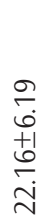 & $\begin{array}{c}0 \\
+ \\
0 \\
+1 \\
+1 \\
\stackrel{+}{0} \\
\leftarrow\end{array}$ & \\
\hline 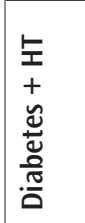 & 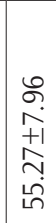 & $\mid \begin{array}{c}\stackrel{\infty}{+} \\
\stackrel{+}{+} \\
\stackrel{+1}{+1} \\
\dot{0} \\
\dot{m}\end{array}$ & 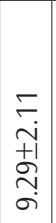 & 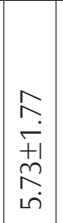 & 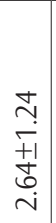 & 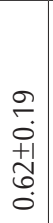 & 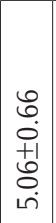 & 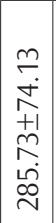 & 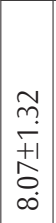 & 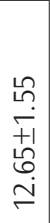 & $\begin{array}{l}\underset{\sim}{\sim} \\
\underset{+1}{+1} \\
\stackrel{\sim}{\sim} \\
\dot{\sim}\end{array}$ & 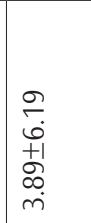 & 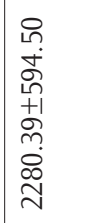 & 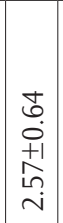 & $\begin{array}{l}\stackrel{0}{\sim} \\
\stackrel{+}{+} \\
+1 \\
\stackrel{+}{+} \\
\stackrel{N}{v}\end{array}$ & 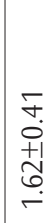 & \\
\hline 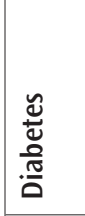 & 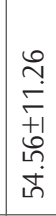 & $\begin{array}{c}0 \\
\infty \\
i \\
+1 \\
0 \\
0 \\
\dot{i} \\
\dot{i}\end{array}$ & 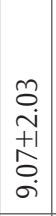 & 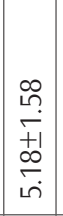 & $\begin{array}{l}\bar{\alpha} \\
0 \\
0+1 \\
\infty \\
\infty \\
i \\
ن\end{array}$ & $\begin{array}{c}\stackrel{0}{1} \\
0 \\
+1 \\
\vdots \\
0 \\
0\end{array}$ & 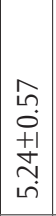 & 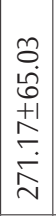 & 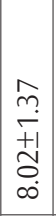 & 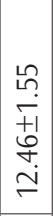 & 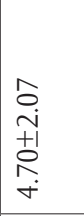 & 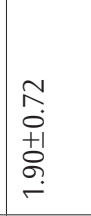 & 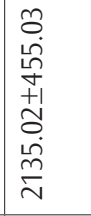 & 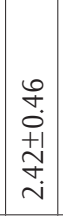 & 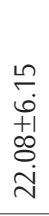 & 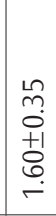 & \\
\hline 일 & 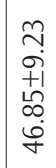 & 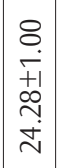 & $\begin{array}{c}b \\
i \\
+1 \\
+1 \\
m \\
\sigma\end{array}$ & $\begin{array}{c}\hat{N} \\
\sim \\
+1 \\
\tilde{N} \\
\hat{N} \\
\end{array}$ & $\begin{array}{l}\text { o. } \\
0 \\
+1 \\
+1 \\
0 \\
\end{array}$ & 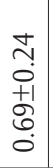 & $\begin{array}{c}8 \\
\dot{0} \\
0 \\
+1 \\
0 \\
\stackrel{1}{1} \\
\end{array}$ & 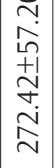 & 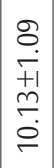 & 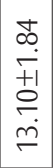 & $\begin{array}{l}\bar{\sigma} \\
i \\
+1 \\
0 \\
0 \\
\dot{+} \\
\end{array}$ & $\begin{array}{c}\stackrel{g}{g} \\
\stackrel{+}{+1} \\
\stackrel{1}{n} \\
\stackrel{i}{i}\end{array}$ & 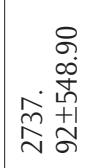 & $\begin{array}{l}\tilde{N} \\
0 \\
0 \\
+1 \\
0 \\
0 \\
i\end{array}$ & 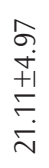 & 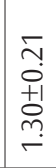 & \\
\hline & 总 & $\grave{\infty}$ & 3 & 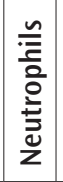 & 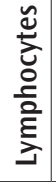 & & 品 & $\bar{a}$ & $\frac{2}{\Sigma}$ & & 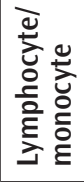 & 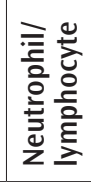 & 主 & 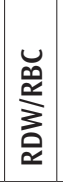 & $\begin{array}{l}\frac{z}{0} \\
\stackrel{\underline{a}}{\underline{\underline{a}}}\end{array}$ & $\sum_{\substack{a \\
\Sigma}}^{\frac{2}{a}}$ & \\
\hline
\end{tabular}


the mean age was statistically significantly higher in diabetes + cardiovascular disease and cardiovascular groups compared to the other subgroups. Similarly, in our study the mean BMI value was statistically significantly higher in the OSAS group compared to the control group. As respiratory symptoms are more common in people with an advanced age and overweight persons, this situation is an expected finding.

It is well-known that MPV is increased with hypoxia (25). Nena et al. (26) reported that MPV and platelet distribution width (PDW) increase in OSAS patients.

OSAS is associated with insulin resistance and impaired glucose tolerance, and induces endothelial dysfunction (27). In our study, both MPV and total platelet mass were significantly higher among OSAS patients compared to the controls. Kanbay et al. (20) demonstrated that MPV levels increased as the severity of OSAS increased, and pointed out that high MPV levels were associated with the prevalence of CVD in OSAS patients (20). In the current study, CVD was found in 15, and both diabetes and CVD in nine OSAS patients. We think that, lower rate of CVD in our study was caused by the inability to measure the severity of OSAS, because the primary objective of our study was to investigate total platelet mass in OSAS patients.

Studies in the literature have reported higher MPV values in OSAS patients compared to the controls and accordingly, it has been argued that MPV value is a diagnostic marker for OSAS especially in patients presenting to emergency departments $(20,25,26,28,29)$.

However, as we mentioned before MPV should be taken into account together with platelet count and not alone. In fact, in the majority of the above mentioned studies, MPV value was high in OSAS patients depending on the severity of the disease, while platelet count has been reported as low according to the number of patients. In the present study, both the mean MPV value and total platelet mass found by MPV x platelet count were statistically significantly lower in OSAS patients compared to the control group. Therefore, unlike the previous studies MPV and/ or total platelet mass is not sufficient for the diagnosis of OSAS, especially in patients presenting to emergency departments with the complaints of fatigue, headache, distractibility and daytime drowsiness, and these patients must be directed to polysomnography.

\section{Study Limitations}

The main limitation of our study is its retrospective design and being conducted in a single center. The effects of geographic areas and climate can on OSAS can be investigated with further studies. However, the number of our patients is relatively high for such a study, which reflects the strong aspect of our study. In addition, this study is the first in the literature investigating not only MPV, but also total platelet mass in OSAS patients. Our results that are contrary to those of the previous studies should be supported with further multi-center comprehensive studies.

\section{Conclusion}

According to the results of this study; MPV and/or total platelet count is not diagnostic, especially in patients presenting to emergency departments with the complaints of fatigue, headache, distractibility and daytime drowsiness. These patients must be directed to polysomnography. However, the high prevalence of comorbidities such as hypertension, DM, CVD and hyperlipidemia is remarkable. Patient groups with these comorbidities can be evaluated for OSAS in future studies. We believe that the results of this study will create a new perspective in the literature in the role of platelet activation in OSAS.

\section{Ethics}

Ethics Committee Approval: Before the beginning, the study protocol was approved by the Local Ethics Committee of Harran University with 11/05/2020 dated and HRU/20.09.09 numbered decision.

Informed Consent: Retrospective study.

Peer-review: Externally peer-reviewed.

\section{Authorship Contributions}

Surgical and Medical Practices: E.S.Ş., Concept: E.S.S., Design: E.S.S., Data Collection or Processing: I.H.Y., Analysis or Interpretation: I.H.Y., Literature Search: I.H.Y., Writing: E.S.Ş.

Conflict of Interest: The authors declare no conlict of interest to disclose.

Financial Disclosure: This study did not receive financial support.

\section{References}

1. McNicholas WT, Bonsigore MR; Management Committee of EU COST ACTION B26. Sleep apnoea as an independent risk factor for cardiovascular disease: current evidence, basic mechanisms and research priorities. Eur Respir J. 2007;29:156-78.

2. Young T, Palta M, Dempsey J, Skatrud J, Weber S, Badr S. The occurrence of sleep-disordered breathing among middle-aged adults. N Engl J Med. 1993;328:1230-5.

3. Young T, Peppard P, Palta M, Hla KM, Finn L, Morgan B, et al. Populationbased study of sleep-disordered breathing as a risk factor for hypertension. Arch Intern Med. 1997;157:1746-52.

4. Rosen CL. Obstructive sleep apnea syndrome (OSAS) in children: diagnostic challenges. Sleep. 1996;19(10 Suppl):274-7.

5. Kheirandish-Gozal L, Kim J, Goldbart AD, Gozal D. Novel pharmacological approaches for treatment of obstructive sleep apnea in children. Expert Opin Investig Drugs. 2013;22:71-85. 
6. American Academy of Sleep Medicine. Obstructive sleep Apnea. Illinois: American Academy of Sleep Medicine 2008.

7. Badimon L, Padró T, Vilahur G. Atherosclerosis, platelets and thrombosis in acute ischaemic heart disease. Eur Heart J Acute Cardiovasc Care. 2012;1:6074.

8. Kamath S, Blann AD, Lip GY. Platelet activation: assessment and quantification. Eur Heart J. 2001;22:1561-71.

9. Malerba M, Olivini A, Radaeli A, Ricciardolo FL, Clini E. Platelet activation and cardiovascular comorbidities in patients with chronic obstructive pulmonary disease. Curr Med Res Opin. 2016;32:885-91.

10. Akyol S, Çörtük M, Baykan AO, Kiraz K, Börekçi A, Şeker T, et al. Mean platelet volume is associated with disease severity in patients with obstructive sleep apnea syndrome. Clinics (Sao Paulo). 2015;70:481-5.

11. Topcuoglu OB, Afsar GC, Sarac S, Oruc O, Kuyucu T. In the absence of comorbidities mean platelet volume is not a severity indicator in OSAS. Eur J Gen Med. 2016;13:134-8.

12. Girgin R, Cinar O, Bulut E, Akduman B, Mungan NA. The Role of the Platelet Mass Index (PMI) as a New Prognostic Factor in Fournier's Gangrene. African Journal of Urology. 2018;24:226-32.

13. Atan D, Sazak Kundi FC, Özcan KM, Dere H. The Relationship Between Platelet Count and Mean Platelet Volume With Obstructive Sleep Apnea Syndrome. Kulak Burun Bogaz Ihtis Derg. 2015;25:289-94.

14. Dikbas 0 , Erten N, Kucuker F, Aksehirli Yilmaz O. Relationship of Potential Inflammatory Markers Namely Neutrophile Lymphocyte ratio and Platelet Lymphocyte ratio With the Severity of Obstructive Sleep Apnea. Turk J Diab Obes/Türk Diyab Obez Derg. 2017;3:125-31.

15. Gong W, Wang X, Fan J, Nie S, Wei Y. Impact of Obstructive Sleep Apnea on Platelet Function Profiles in Patients With Acute Coronary Syndrome Taking Dual Antiplatelet Therapy. J Am Heart Assoc. 2018;7:e008808.

16. Rechtschaffen A, Kales A, eds. A manual of standardized terminology, techniques and scoring system of sleep stages in human subjects. Los Angeles: Brain Information Service/Brain Research Institute, University of California; 1968.

17. Martin JF, Trowbridge EA, Salmon G, Plumb J. The biological significance of platelet volume: its relationship to bleeding time, platelet thromboxane B2 production and megakaryocyte nuclear DNA concentration. Thromb Res. 1983;32:443-60.

18. Giles H, Smith RE, Martin JF. Platelet glycoprotein IIb-IIla and size are increased in acute myocardial infarction. Eur J Clin Invest. 1994;24:69-72.

19. Jakubowski JA, Thompson CB, Vaillancourt R, Valeri CR, Deykin D. Arachidonic acid metabolism by platelets of differing size. Br J Haematol. 1983;53:50311.

20. Kanbay A, Tutar N, Kaya E, Buyukoglan H, Ozdogan N, Oymak FS, et al. Mean platelet volume in patients with obstructive sleep apnea syndrome and its relationship with cardiovascular diseases. Blood Coagul Fibrinolysis. 2013;24:532-6

21. Varol E, Ozturk O, Gonca T, Has M, Ozaydin M, Erdogan D, et al. Mean platelet volume is increased in patients with severe obstructive sleep apnea. Scand J Clin Lab Invest. 2010;70:497-502.

22. Chu SG, Becker RC, Berger PB, Bhatt DL, Eikelboom JW, Konkle B, et al. Mean platelet volume as a predictor of cardiovascular risk: a systematic review and meta-analysis. J Thromb Haemost. 2010 ;8:148-56.

23. Bath PM, Butterworth RJ. Platelet size: measurement, physiology and vascular disease. Blood Coagul Fibrinolysis. 1996;7:157-61.

24. Phillips BG, Somers VK. Sleep disordered breathing and risk factors for cardiovascular disease. Curr Opin Pulm Med. 2002;8:516-20.

25. Parish JM, Somers VK. Obstructive sleep apnea and cardiovascular disease. Mayo Clin Proc. 2004;79:1036-46.

26. Nena E, Papanas N, Steiropoulos P, et al. Mean platelet volume and platelet distribution width in nondiabetic subjects with obstructive sleep apnea syndrome: new indices of severity? Platelets. 2012;23:447-54.

27. Laaban JP, Mounier L, Roque d'Orbcastel O, et al. Cardiovascular risk factors in men and women with obstructive sleep apnoea syndrome. Respir Med. 2010;104:1063-8.

28. Kamio K, Ono Y, Kamiya U, et al. Nihon Kokyuki Gakkai Zasshi. 2002;40: 473-7.

29. Kondo Y, Kuwahira I, Shimizu M, et al. Significant relationship between platelet activation and apnea-hypopnea index in patients with obstructive sleep apnea syndrome. Tokai J Exp Clin Med. 2011;36:79-83. 